\title{
Thrombin-induced lysosomal exocytosis in human platelets is dependent on secondary activation by ADP and regulated by endothelial-derived substances
}

\author{
Anna Södergren, Ann-Charlotte Svensson Holm, Sofia Ramström, Eva Lindström, \\ Magnus Grenegård and Karin Öllinger
}

\author{
Linköping University Post Print
}

\section{Tweet}

N.B.: When citing this work, cite the original article.

This is an electronic version of an article published in:

Anna Södergren, Ann-Charlotte Svensson Holm, Sofia Ramström, Eva Lindström, Magnus Grenegård and Karin Öllinger, Thrombin-induced lysosomal exocytosis in human platelets is dependent on secondary activation by ADP and regulated by endothelial-derived substances, 2016, Platelets, (27), 1, 86-92.

Platelets is available online at informaworld ${ }^{\mathrm{TM}}$ :

http://dx.doi.org/10.3109/09537104.2015.1042446

Copyright: Taylor \& Francis: STM, Behavioural Science and Public Health Titles http://www.tandf.co.uk/journals/default.asp

Postprint available at: Linköping University Electronic Press

http://urn.kb.se/resolve?urn=urn:nbn:se:liu:diva-125318 
Thrombin-induced lysosomal exocytosis in human platelets is dependent on secondary activation by ADP and regulated by endothelial-derived substances

\author{
Anna L. Södergren ${ }^{1 *}$, Ann-Charlotte B. Svensson Holm ${ }^{*}$, Sofia Ramström ${ }^{1}$, Eva G. \\ Lindström $^{2}$, Magnus Grenegård ${ }^{2,3}$, Karin Öllinger ${ }^{1}$ \\ ${ }^{1}$ Department of Clinical and Experimental Medicine, Faculty of Health Sciences, Linköping University, \\ Linköping, Sweden \\ ${ }^{2}$ Department of Medical and Health Sciences, Faculty of Health Sciences, Linköping University, Linköping, \\ Sweden \\ ${ }^{3}$ Department of Clinical Medicine, School of Health Sciences, Örebro University, Örebro, Sweden \\ * these authors contributed equally to the study
}

Running title: Lysosomal exocytosis in human platelets

Correspondence:

Karin Öllinger, Experimental Pathology, Department of Clinical and Experimental Medicine, Faculty of Health Sciences, Linköping University, SE-581 85 Linköping, Sweden Tel: +46-10-1033261

E-mail: karin.ollinger@liu.se

Keywords: ADP receptors, Endothelium, Exocytosis, Lysosome, Platelet physiology, Protease activated receptors (PAR), Thrombin 


\begin{abstract}
Exocytosis of lysosomal contents from platelets has been speculated to participate in e.g. clearance of thrombi and vessel wall remodelling. The mechanisms that regulate lysosomal exocytosis in platelets are, however, still unclear. The aim of this study was to identify the pathways underlying platelet lysosomal secretion and elucidate how this process is controlled by platelet inhibitors. We found that high concentrations of thrombin induced partial lysosomal exocytosis as assessed by analysis of the activity of released $\mathrm{N}$-acetyl- $\beta$ glucosaminidase (NAG) and by identifying the fraction of platelets exposing the lysosomal associated membrane protein (LAMP)-1 on the cell surface by flow cytometry. Stimulation of thrombin receptors PAR1 or PAR4 with specific peptides was equally effective in inducing LAMP-1 surface expression. Notably, lysosomal exocytosis in response to thrombin was significantly reduced if the secondary activation by ADP was inhibited by the P2 $\mathrm{Y}_{12}$ antagonist cangrelor, while inhibition of thromboxane $A_{2}$ formation by treatment with acetylsalicylic acid was of minor importance in this regard. Moreover, the NO-releasing drug S-nitroso-N-acetyl penicillamine (SNAP) or the cyclic AMP-elevating eicosanoid prostaglandin $\mathrm{I}_{2}\left(\mathrm{PGI}_{2}\right)$ significantly suppressed lysosomal exocytosis. We conclude that platelet inhibitors that mimic functional endothelium such as $\mathrm{PGI}_{2}$ or $\mathrm{NO}$ efficiently counteract lysosomal exocytosis. Furthermore, we suggest that secondary release of ADP and concomitant signaling via PAR-1/4- and $\mathrm{P}_{2} \mathrm{Y}_{12}$ receptors is important for efficient platelet lysosomal exocytosis by thrombin.
\end{abstract}




\section{Introduction}

Upon activation, platelets are able to release contents from three types of granules; $\alpha$-granules, dense granules and lysosomes. $\alpha$-Granules contain a vast variety of proteins involved in coagulation, adhesion, inflammation, cell growth and host defence [1]. The dense granules contain small molecules including $\mathrm{Ca}^{2+}$, ADP, ATP and serotonin, which are involved in secondary activation and recruitment of additional platelets [2]. Lysosomes are ubiquitous intracellular organelles with an acidic interior containing a large number of hydrolytic enzymes. In contrast to the rather simplified view of lysosomes as waste bags for damaged macromolecules and organelles, today we recognize lysosomes as advanced organelles that are involved in many cellular processes and act as crucial regulators of cell homeostasis [3]. Furthermore, lysosomes are known to play a role in both apoptosis and necrosis [4-6]. The lysosomal membrane is traversed by highly glycosylated transmembrane proteins including the lysosomal associated membrane proteins (LAMPs). Febbraio and Silverstein showed that the luminal part of LAMP-1 is exposed on the platelet surface upon platelet activation, indicating that lysosomes are secreted under certain conditions [7]. An increased release of the lysosomal enzyme platelet acid phosphatase was also reported in response to several platelet agonists including collagen, thrombin and immune complexes [8]. Following platelet activation, dense granules are the most rapidly secreted followed by $\alpha$-granules and lysosomes. While $\alpha$-granules and dense granules can reach close to $100 \%$ secretion, lysosomes do not show complete secretion even upon stimulation with strong agonists $[2,9,10]$.

Recently it was shown that lysosomal exocytosis in platelets is dependent on syntaxin binding protein 5, which interacts with the secretory complex proteins [11]. In human volunteers inflicted with a wound to estimate bleeding time, the activated platelets showed surface expression of LAMP-1, LAMP-2 and LIMP (LAMP-3, CD63), indicating that lysosomal exocytosis occur "in vivo" [9]. The role of lysosomal exocytosis in platelets is, 
however, still unclear. It has been suggested to be involved in the clearance of thrombi, vessel wall remodelling and modulation of platelet membrane proteins [9, 12]. Moreover lysosomal exocytosis could play a role in adhesion, since expression of LAMP-l is observed on cells with increased adhesive capacity, such as transformed cells of high metastatic potential, and interestingly, on embryonic cells [13]. Exocytosis of proteolytic lysosomal enzymes may also be beneficial for host defence [14]. Noteworthy, Coorssen et al [15] found that a $\mathrm{Ca}^{2+}$-triggered exocytosis of intracellular vesicles (later suggested to be lysosomes [16]) led to a 20-30\% increase in the surface area of Chinese hamster ovary cells.

Adhesion and aggregation of platelets at the site of vascular injury is essential for hemostasis and thrombosis and is mediated through receptors and release/production of different mediators. The major platelet agonist thrombin activates human platelets via the protease activated receptors (PAR) 1 and 4. Thrombin binds to the PARs and cleaves the N-terminal of the receptors, autoactivation then occurs when the new exposed $\mathrm{N}$-terminal binds to the active site of the receptor $([17,18]$ and reviewed in $[19])$. Furthermore, the effect of platelet activators is enhanced through positive feedback loops: e.g. the formation of thromboxane $A_{2}\left(\mathrm{TXA}_{2}\right)$ and the secretion of ADP [20].

Prostaglandin $\mathrm{I}_{2}$ ( $\mathrm{PGI}_{2}$, also called prostacyclin) and nitric oxide (NO) are molecules physiologically important for suppressing platelet activation and platelet-vessel wall interaction. They are secreted from the vascular endothelium and act in a paracrine manner [21]. $\mathrm{PGI}_{2}$ and NO do not only inhibit platelet activation, they also synergistically reverse platelet aggregation [22] which is important to maintain blood fluidity and minimize thrombus formation.

In this study, we have investigated the kinetics and magnitude of lysosomal exocytosis from human platelets and its regulation. We found that only a subpopulation of platelets responds to activation by lysosomal exocytosis as detected by LAMP-1 exposure on the platelet surface. 
Lysosomal exocytosis, determined as N-acetyl- $\beta$-glucosaminidase (NAG) activity or LAMP-1 exposure, was dependent on secondary activation by ADP and was controlled by the endothelial derived inhibitors $\mathrm{NO}$ and $\mathrm{PGI}_{2}$. 


\section{Materials and methods}

\section{Chemicals and antibodies}

The chemicals used were as follows: S-Nitroso-N-acetyl-DL-penicillamine (SNAP), apyrase grade III, prostaglandin $\mathrm{I}_{2}$ (PGI $)$, human thrombin, 4-methylumbelliferyl N-acetyl-D- $\beta$ glucosaminide, acetylsalicylic acid (ASA), ADP and chemicals for the HEPES buffer (pH 7.4, $137 \mathrm{mM} \mathrm{NaCl}, 2.7 \mathrm{mM} \mathrm{KCl}, 1 \mathrm{mM} \mathrm{MgCl}_{2}$, $5.6 \mathrm{mM}$ glucose, $1 \mathrm{~g} / \mathrm{l} \mathrm{BSA}, 20 \mathrm{mM}$ HEPES) supplemented with $2.5 \mathrm{mM} \mathrm{CaCl}_{2}$, were from Sigma Chemical Co. (St. Louis, MO, USA). Cangrelor was a kind gift from the Medicines Company (Parsippany, NJ, USA), PAR1activating peptide (AP) with the amino acid sequence SFLLRN, PAR4-AP with the sequence AYPGKF and the peptide GPRP were from JPT Peptide Technologies GmbH (Berlin, Germany). Recombinant hirudin (lepirudin, Refludan ${ }^{\circledR}$ ) was from Aventis (Strasbourg, France). For flow cytometric analysis the following antibodies were used: anti-GPIIb-Rphycoerythrin (PE) (CD41a; clone: H1P8, 25 ng/ml), anti-P-selectin-PE-Cy5 (CD62P; clone: AK-4, $1 \mu \mathrm{g} / \mathrm{ml}$ ), anti-LAMP-1 (CD107a, clone: H4A3 conjugated to FITC, 4.2 or $8.3 \mu \mathrm{g} / \mathrm{ml}$ or PE-Cy5, $0.5 \mu \mathrm{g} / \mathrm{ml}$, all from Becton Dickinson (Franklin Lakes, NJ, USA). The LAMP-1 clone is directed to the luminal part of LAMP-1, which upon exocytosis relocates to the cell surface [14].

\section{Blood collection}

Venous blood was collected from healthy volunteers into Vacutainer ${ }^{\circledR}$ tubes (Becton Dickinson) or obtained from the local blood donation centre. Heparinised blood (11-17 U/ml) was obtained for isolation of platelets from blood bags as this was a part of the local procedure, and for experiments with PAR-APs to prevent any formation of active thrombin that could have interfered with the results. Control experiments confirmed that heparin itself caused no platelet activation response in these settings. For flow cytometry experiments with thrombin activation, 
citrate anticoagulation $(0.105 \mathrm{M})$ was used. Blood collection procedures were approved by the Local Ethics Committee in Linköping and all blood donors gave their informed consent.

\section{Platelet isolation}

Heparinized blood was mixed with acid citrate/dextrose solution (ACD, 5:1) and centrifuged (220 $\mathrm{g}$ for $20 \mathrm{~min}$ ) to obtain platelet-rich plasma (PRP). The supernatant was collected and supplemented with apyrase $(1 \mathrm{U} / \mathrm{ml})$ and PGI $2(0.5 \mu \mathrm{g} / \mathrm{ml})$, and centrifuged at $480 \mathrm{~g}$ for $20 \mathrm{~min}$. The platelet pellet was gently washed and resuspended in calcium free Krebs-Ringer glucose phosphate buffer (KRG) and counted.

\section{Inhibitors}

When indicated, platelets were pretreated with ASA (100 $\mu \mathrm{M}, 15 \mathrm{~min})$, cangrelor $(5 \mu \mathrm{M}, 5$ min), SNAP (10 or $30 \mu \mathrm{M} ; 2 \mathrm{~min})$, $\mathrm{PGI}_{2}(1 \mu \mathrm{M} ; 2 \mathrm{~min})$ or the solvent DMSO (final concentration $0.1-0.3 \%$ ). None of these substances had any effect on lysosomal exocytosis when added alone (data not shown).

Analysis of lysosomal exocytosis by $N$-acetyl- $\beta$-glucosaminidase activity The platelet suspension ( $3 \times 10^{8} / \mathrm{ml}$ ) was incubated with $1 \mathrm{mM} \mathrm{CaCl}_{2}$ for 5 min at $37^{\circ} \mathrm{C}$. Platelets were then stimulated with thrombin for 1 or 5 min after which activation was stopped by addition of ACD (1/100 dilution), hirudin (2 U/ml) and PGI $2(0.5 \mu \mathrm{g} / \mathrm{ml})$, followed by centrifugation at $800 \mathrm{~g}$ for $10 \mathrm{~min}$. The supernatants were collected and incubated at $37^{\circ} \mathrm{C}$ for 40 min in $0.2 \mathrm{M}$ citrate buffer (pH 4.5) containing 0.8 mM 4-methylumbelliferyl N-acetyl-D$\beta$-glucosaminide [23]. Fluorescence from methylumbelliferyl released by the enzyme was determined using a Victor Wallac multilabel plate reader (PerkinElmer) at $\lambda_{\text {ex }} 356$ and $\lambda_{\text {em }} 444$ 
nm. NAG release was expressed as a fraction of the total NAG activity, which was obtained by lysis of all platelets in $1 \%$ Triton-X-100.

Analysis of LAMP-1 and P-selectin by flow cytometry

Citrated whole blood or PRP (obtained by centrifugation at $220 \mathrm{~g}, 20 \mathrm{~min}$ ) was diluted (final dilution 1:5) in a mastermix, containing 2 mM GPRP to avoid fibrin polymerisation, thrombin ( 0.3 or $1 \mathrm{U} / \mathrm{ml}$, final concentrations) or ADP $(5 \mu \mathrm{M})$ and, when indicated, inhibitors or vehicle. After 1 or 10 minutes of activation at room temperature (RT), a stop solution containing hirudin (final concentration $4 \mathrm{U} / \mathrm{ml}$ ) and antibodies; anti-GPIIb, anti-P-selectin and anti-LAMP-1-FITC was added. After an additional 10 minutes of incubation at RT, samples were diluted 1:20 in HEPES buffer and surface expression of LAMP-1 and P-selectin was analysed on a Gallios ${ }^{\mathrm{TM}}$ flow cytometer (Beckman Coulter, Fullerton, CA, USA). LAMP-1 was chosen as the lysosomal marker, as both LAMP-2 and LIMP (CD63) have been shown to be present in the membrane of dense granules as well [24, 25].

To study activation induced by stimulation of the platelet thrombin receptors PAR1 and PAR4 separately and in combination, an experimental system of low, medium or high level of platelet activation was designed. Thus, agonist concentrations were selected to achieve a Pselectin expression level of approximately 20\%, 50\% and 100\% respectively (Supplementary Figure S1). Final concentrations were PAR1-AP: 2.5, 5 or $30 \mu \mathrm{M}$, and PAR4-AP: 25, 50 or 150 $\mu \mathrm{M}$. Heparinized whole blood (12 $\mu \mathrm{l})$ was added to a buffer $(104 \mu \mathrm{l})$ containing agonist and 29 $\mu \mathrm{l}$ of this mixture was transferred, directly or after $45 \mathrm{~min}$, to $7.5 \mu \mathrm{l}$ of antibody solution containing anti-GPIIb and anti-LAMP-1-PE-Cy5. Samples were incubated for 10 minutes (yielding total stimulation of 10 and 55 minutes, respectively) at RT before diluting 1:20 in HEPES buffer and analysing on a Coulter Epics XL.MCL flow cytometer (Beckman Coulter). 
The flow cytometry analysis was performed on 10000 platelets per sample using log forward scatter (FS) and log FL2, where FL2 corresponded to detection of GPIIb-PE, i.e. all plateletderived particles. All data was further analysed using the Kaluza ${ }^{\mathrm{TM}}$ software (Beckman Coulter). To determine the expression of different markers, gates were placed using isotype control antibodies with matching fluorescence intensity and concentration as a reference to set the background to 1-2\%. Samples showing more than $15 \%$ spontaneous expression of P-selectin were excluded from analysis.

Analysis of platelet aggregation and ATP release

Platelet aggregation was measured by analysis of changes in light transmission using a Chronolog Dual Channel lumi-aggregometer (Chrono-log Corporation, Haverston, PA, USA) according to the instruction of the manufacturer. Before each assay, the platelet suspension (3 $\mathrm{x} 10^{8} / \mathrm{ml}$ ) containing $1 \mathrm{mM} \mathrm{CaCl}_{2}$ was incubated in the aggregometer for $5 \mathrm{~min}$ at $37^{\circ} \mathrm{C}$ under continuous stirring at $900 \mathrm{rpm}$. Thrombin was added and the aggregation response was followed for $10 \mathrm{~min}$, which corresponded to maximal aggregation. Aggregation is expressed as percentage light transmission compared with KRG alone (=100\%). In parallel, ATP secretion was measured using the Chrono-Lume ${ }^{\circledR}$ kit (Chrono-log Corporation) and the ATP concentration was calculated by an exogenously added ATP standard.

\section{Statistical analysis}

Data are expressed as means \pm standard error of the mean (SEM). For statistical analysis paired t-tests were used when two groups were compared. When several groups were compared, oneway or repeated measures ANOVA followed by Dunnett's multiple comparison test against control or Bonferroni's multiple comparison tests was used. A p-value $<0.05$ was considered significant. Data were analysed using GraphPad Prism ${ }^{\mathrm{TM}}$ (GraphPad Software, San Diego, CA). 


\section{Results}

Lysosomal exocytosis was analyzed as activity of the lysosomal enzyme NAG in the supernatant, representing total lysosomal exocytosis from the whole platelet population, and as surface expression of the lysosomal protein LAMP-1 by flow cytometry to estimate the fraction of individual platelets responding by lysosomal exocytosis. Thrombin caused release of NAG in a dose-dependent manner after stimulation for $5 \mathrm{~min}$ (Figure 1A). The thrombin concentration was set to $1 \mathrm{U} / \mathrm{ml}$ for the experiments, since stimulation with $5 \mathrm{U} / \mathrm{ml}$ did not cause higher release of NAG. Study of the kinetics revealed that NAG release reached a plateau already after 1 min of stimulation and that prolonged stimulation up to 30 min did not enhance the activity of NAG in the supernatant (Figure 1B). Flow cytometry further supported that lysosomal exocytosis was quick, reaching $33 \pm 2 \%$ at 1 min stimulation with $1 \mathrm{U} / \mathrm{ml}$ thrombin. However, a time dependent increase of LAMP-1 surface exposure was detected especially at lower concentrations of thrombin (Figure 1C).

Next we studied the participation of the platelet thrombin receptors PAR1 and PAR4 in lysosomal exocytosis after $10 \mathrm{~min}$ of stimulation using flow cytometry. Agonist concentrations were chosen to generate low (approx. 20\%), medium (approx. 50\%) and high (approx. 100\%) expression of P-selectin as illustrated in Supplementary Figure S1. LAMP-1 expression levels, significantly higher than in control samples, were achieved with a combination of PAR1-AP and PAR4-AP at all concentrations, whereas PAR1-AP and PAR4-AP alone resulted in an increased LAMP-1 expression at high agonist concentrations (Figure 2). Prolonging the activation time to 55 min raised the LAMP-1 surface expression to approximately $45 \%$ while control samples remained at the same level as after 10 min (Supplementary figure S2). No differences in LAMP-1 expression were detected between platelet activation with PAR1-AP and PAR4-AP at corresponding levels of activation and therefore the remaining experiments were carried out using thrombin. 
To investigate the regulation of thrombin-induced lysosomal exocytosis in platelets, we inhibited cyclooxygenase using ASA and the ADP-receptor P2Y 12 by addition of cangrelor. We found a significant inhibition of NAG release and LAMP-1 surface expression when cangrelor or a combination of the inhibitors was used, while ASA alone did not show a significant effect (Figure $3 \mathrm{~A}$ and $\mathrm{B}$ ). Prolonging the thrombin stimulation to 5 min (NAG release) or $10 \mathrm{~min}$ (LAMP-1 surface expression) did not alter the inhibitory effect of the inhibitors (Supplementary Figure S3). As cangrelor prevents the secondary ADP-induced activation of platelets, we also tested the effect of ADP alone. Activation with ADP (5 $\mu \mathrm{M}, 10$ minutes) resulted in $16 \pm 3 \%$ LAMP-1 exposure as compared to $1.5 \pm 0.2 \%$ in control samples and $60 \pm 2 \%$ in response to thrombin ( $1 \mathrm{U} / \mathrm{ml}, 10 \mathrm{~min})$ (mean $\pm \mathrm{SEM}, \mathrm{n}=11)$. The corresponding expression of P-selectin was $95 \pm 1$ (ADP), $11 \pm 2$ (control) and 99 \pm 0 (thrombin). Platelet aggregation and ATP-secretion in response to thrombin were not affected by either cangrelor or ASA, whereas a slight but significant decrease in P-selectin expression was observed in the presence of cangrelor (1 min; Figure 3C-E).

Addition of the cyclic AMP (cAMP)-elevating eicosanoid PGI2 significantly prevented lysosomal exocytosis from platelets stimulated with thrombin (Figure 4A and B). Addition of SNAP prevented NAG release from isolated platelets (Figure 4A), but did not cause any reduction of LAMP-1 surface expression in diluted whole blood (Supplementary figure S4A). We hypothesized the lack of effect of SNAP in diluted whole blood samples to be due to scavenging of NO by hemoglobin. These experiments were therefore repeated using PRP, where $10 \mu \mathrm{M}$ SNAP efficiently reduced LAMP-1 surface expression in response to thrombin (Figure 4C), with no further reduction observed at $30 \mu \mathrm{M}$ (Supplementary figure S4B). The same inhibitory effect was observed also after 5 min (NAG release) or 10 min (LAMP-1 expression) of stimulation with thrombin (Supplementary figure S5). Thrombin-induced platelet aggregation and ATP secretion was significantly inhibited by SNAP but less affected 
by $\mathrm{PGI}_{2}$, whereas P-selectin expression was reduced by both $\mathrm{PGI}_{2}$ and SNAP (in PRP)

(Figure 4D-G). 


\section{Discussion}

Our data show that high doses of thrombin or combined PAR1/4-AP only provoke partial lysosomal release, even at conditions where all platelets show $\alpha$-granule release (P-selectin exposure). This is in accordance with previous findings [9, 10, 26]. Thrombin-induced NAG release was around $25 \%$ of total NAG activity, while the fraction of LAMP-1 positive platelets was $35-45 \%$ at a similar stimulation. The NAG release was rapid and no increase over time was found, similar to what was reported by Holmsen et al [26], whereas LAMP-1 exposure increased slowly at extended stimulation. One potential explanation to this discrepancy may be that some NAG activity is lost during extended incubation times. Thrombin activates human blood platelets through binding, cleavage and activation of PAR1- and PAR4 receptors. In our study, stimulation with PAR1-AP or PAR4-AP induced similar levels of LAMP-1 expression upon stimulation, demonstrating that both receptors can be used to induce lysosomal exocytosis. However, according to our flow cytometric analysis, only a fraction of the platelets responded to thrombin receptor activation with lysosomal secretion, suggesting that only a sub-population of platelets will respond with lysosomal release. Whether lysosomal exocytosis is the only characteristic shared within this sub-population of platelets remains to be determined.

It is well established that the effect of the primary platelet activators is enhanced by positive feedback loops. Release of TXA 2 and ADP are the most characterized secondary autocrine activators and we found that pre-treatment of platelets with the ADP P2Y 12 receptor antagonist cangrelor significantly decreased thrombin-induced lysosomal exocytosis. A reduction in NAG release has previously been found upon pre-incubation of platelets with the ADP-removing system, creatine phosphate/creatine phosphokinase [27]. Moreover, we observed that thrombin-induced aggregation was not reduced by cangrelor. This is in line with previous findings showing that aggregation induced by high doses of thrombin is independent of the secondary ADP release [28]. Thrombin-induced PAR1 and PAR4 activation 
leads to long-lasting $\mathrm{G \alpha}_{12 / 13^{-}}$and $\mathrm{G} \alpha_{\mathrm{q}}$-mediated intracellular signaling whereas the secondary release of ADP and activation of $\mathrm{P}_{2} \mathrm{Y}_{12}$ contributes with $\mathrm{G}_{\mathrm{i}}$-coupled signaling [29]. Our results suggests that concomitant activation of $\mathrm{G} \alpha_{12 / 13^{-}}, \mathrm{G} \alpha_{\mathrm{q}^{-}}$and $\mathrm{G} \alpha_{\mathrm{i}^{-}}$coupled receptors is important for efficient lysosomal secretion. In line with this, activation by ADP alone resulted in a modest but clear LAMP-1 expression whereas full P-selectin expression was seen. This further supports the notion that the signaling pathway synergy is important for efficient lysosomal exocytosis, while this is not required for release of $\alpha$-granules. This is partly consistent with previous studies [7, 30], reporting that ADP is a weak inducer of lysosomal exocytosis. However, due to differences in methodology and presentation of data, a complete comparison of the results is not possible. In this study, inhibition of TXA 2 synthesis by ASA did not induce a significant alteration of lysosomal exocytosis. In common with PARs, the predominate $\mathrm{TXA}_{2}$ surface receptor $\mathrm{TP} \alpha$ initiates $\mathrm{G} \alpha_{\mathrm{q}}$-mediated signaling in platelets [29]. This overlap in signaling pathways may explain why TXA 2 plays no role when thrombin is present. This is in accordance with experiments performed by Vorland and Holmsen who found no effect on lysosomal secretion when using the specific TXA 2 inhibitor SQ 29.548 in thrombin-stimulated platelets [27] and Chronos et al who compared the exposure of CD63 and P-selectin in healthy subjects before and after ingestion of ASA [31]. McKenzie et al reported that the spontaneous expression of LAMP-1, LIMP and P-selectin was reduced in platelets treated with ASA in vitro [32]. In contrast, we did not observe any effect of ASA on spontaneous lysosomal or $\alpha$-granule exocytosis. However, as the spontaneous level of LAMP1 and P-selectin expressions in both our and McKenzies studies are low (less than $10 \%$ of the platelets were positive for P-selectin), and the observed differences small (approximately 2\% in McKenzie’s study and 1\% in our experiments), we believe that these discrepancies may as well be due to slight differences in pre-analytical handling. Either way the physiological relevance of such small variations can be questioned. Taken together, we conclude that the 
$\mathrm{ADP} / \mathrm{P}_{2} \mathrm{Y}_{12}$ receptor signaling pathway is important for lysosomal exocytosis from thrombinactivated platelets, whereas $\mathrm{TXA}_{2}$ signaling is of minor importance.

There is a large body of evidence that endothelium-derived molecules effectively inhibit human platelets [21]. We found that treatment with $\mathrm{PGI}_{2}$ resulted in decreased lysosomal and $\alpha$-granule exocytosis. In addition, $\mathrm{PGI}_{2}$ showed a slight, although not significant, inhibitory effect on thrombin-induced aggregation and dense granule secretion. ADP signalling through $\mathrm{P} \mathrm{Y}_{12}$ and $\mathrm{G} \alpha_{\mathrm{i}}$ causes a decrease in cAMP levels $[33,34]$ and this is opposed by PGI 2 signalling via $G \alpha_{s}$, which instead causes an increase in the cAMP levels. It is also well known that the $\mathrm{PGI}_{2}$ /cAMP signalling pathway inhibits the agonist-induced rise in intracellular $\mathrm{Ca}^{2+}$ [21]. As lysosomal exocytosis is dependent on intracellular $\mathrm{Ca}^{2+}[14,35]$, it is likely that inhibition of PAR-induced $\mathrm{Ca}^{2+}$ mobilization and subsequent ADP release represent one key target for the $\mathrm{PGI}_{2} / \mathrm{cAMP}$ signalling pathway.

In the present study, the NO-donor SNAP caused a profound reduction in platelet aggregation and prevented secretion of $\alpha$ - and dense granules as well as lysosomes. This was true even at the relatively high thrombin concentrations used in these experiments. It is notable that SNAP did not inhibit lysosomal release in diluted whole blood experiments, but had a pronounced effect in platelet-rich plasma and isolated platelet suspensions. The best available explanation is the presence of hemoglobin, which is an effective scavenger of NO, However, in vivo, the hemoglobin effect would normally be prevented by the formation of a red blood cell-free zone close to the endothelium [36]. Platelet inhibition by NO increases the levels of cGMP, which leads to a marked decrease in intracellular $\mathrm{Ca}^{2+}$ [37]. This implies that inhibition of granule exocytosis and aggregation by $\mathrm{NO}$ and $\mathrm{PGI}_{2}$ may share a similar mechanism, which includes a reduction in secondary activation by decreased ADP release and inhibition of $\mathrm{Ca}^{2+}$-mobilization following PAR activation. From a broader perspective, our 
results indicate that endothelial platelet inhibitors such as $\mathrm{NO}$ and $\mathrm{PGI}_{2}$ easily can control platelet lysosomal secretion.

In conclusion, our study shows that lysosomal exocytosis occurs in a fraction of the platelets upon stimulation of the thrombin receptors PAR1 or PAR4, and that it involves functional positive feedback loops in terms of ADP release and activation of the $\mathrm{P} \mathrm{Y}_{12}$ receptor. We also conclude that lysosomal release, as well as other platelet activation events can be efficiently controlled by physiologically important platelet inhibitors such as NO and $\mathrm{PGI}_{2}$, even at relatively high thrombin concentrations, demonstrating the importance of a functional endothelium in the control of platelet granule release.

\section{Declaration of Interest}

This work was supported by grants from the County Council of Östergötland and the Swedish Research Council. The authors declare no competing financial interests.

Concept and design: MG, SR, KÖ; experimental design, experimental execution and analysis of data A-CBSH and ALS; Data interpretation and writing of manuscript MG, SR, KÖ, EGL, A-CBSH and ALS. All authors have approved the final version of the manuscript. 


\section{References}

1. Coppinger JA, Cagney G, Toomey S, Kislinger T, Belton O, McRedmond JP, Cahill DJ, Emili A, Fitzgerald DJ, Maguire PB. Characterization of the proteins released from activated platelets leads to localization of novel platelet proteins in human atherosclerotic lesions. Blood 2004;103:2096-2104.

2. Rendu F, Brohard-Bohn B. The platelet release reaction: Granules' constituents, secretion and functions. Platelets 2001;12:261-273.

3. Appelqvist H, Waster P, Kagedal K, Ollinger K. The lysosome: From waste bag to potential therapeutic target. J Mol Cell Biol 2013;5:214-226.

4. Jackson SP, Schoenwaelder SM. Procoagulant platelets: Are they necrotic? Blood 2010;116:2011-2018.

5. Repnik U, Stoka V, Turk V, Turk B. Lysosomes and lysosomal cathepsins in cell death. Biochim Biophys Acta 2012;1824:22-33.

6. Kagedal K, Johansson U, Ollinger K. The lysosomal protease cathepsin d mediates apoptosis induced by oxidative stress. FASEB J 2001;15:1592-1594.

7. Febbraio M, Silverstein RL. Identification and characterization of lamp-1 as an activation-dependent platelet surface glycoprotein. J Biol Chem 1990;265:18531-18537.

8. Polasek J. Lysosomal concept of platelet secretion--revisited. Eur J Haematol Suppl 1989;50:3-24.

9. Ciferri S, Emiliani C, Guglielmini G, Orlacchio A, Nenci GG, Gresele P. Platelets release their lysosomal content in vivo in humans upon activation. Thromb Haemost 2000;83:157-164.

10. Jonnalagadda D, Izu LT, Whiteheart SW. Platelet secretion is kinetically heterogeneous in an agonist-responsive manner. Blood 2012;120:5209-5216.

11. Ye S, Huang Y, Joshi S, Zhang J, Yang F, Zhang G, Smyth SS, Li Z, Takai Y, Whiteheart SW. Platelet secretion and hemostasis require syntaxin-binding protein STXBP5. J Clin Invest. 2014;124:4517-28. 12. Emiliani C, Ciferri S, Mencarelli S, Mezzasoma AM, Momi S, Orlacchio A, GreseleP. Defective platelet beta-n-acetyl hexosaminidase content and release in chronic myeloproliferative disorders. Platelets 2006;17:20-29.

13. Lippincott-Schwartz J, Fambrough DM. Lysosomal membrane dynamics: Structure and interorganellar movement of a major lysosomal membrane glycoprotein. J Cell Biol 1986;102:1593-1605.

14. Reddy A, Caler EV, Andrews NW. Plasma membrane repair is mediated by ca(2+)regulated exocytosis of lysosomes. Cell 2001;106:157-169.

15. Coorssen JR, Schmitt H, Almers W. Ca2+ triggers massive exocytosis in chinese hamster ovary cells. EMBO J 1996;15:3787-3791.

16. Rodriguez A, Webster P, Ortego J, Andrews NW. Lysosomes behave as ca2+regulated exocytic vesicles in fibroblasts and epithelial cells. J Cell Biol 1997;137:93-104.

17. Vu TK, Hung DT, Wheaton VI, Coughlin SR. Molecular cloning of a functional thrombin receptor reveals a novel proteolytic mechanism of receptor activation. Cell 1991;64:1057-1068.

18. Rasmussen UB, Vouret-Craviari V, Jallat S, Schlesinger Y, Pages G, Pavirani A, Lecocq JP, Pouyssegur J, Van Obberghen-Schilling E. Cdna cloning and expression of a hamster alpha-thrombin receptor coupled to ca2+ mobilization. FEBS Lett 1991;288:123-128.

19. Brass LF. Thrombin and platelet activation. Chest 2003;124:18S-25S.

20. Freedman JE. Molecular regulation of platelet-dependent thrombosis. Circulation 2005;112:2725-2734.

21. Beaulieu LM, Freedman JE. Inhibition of platelet function by the endothelium. In: Michelson ADs. Platelets 
3rd ed. Place: Elsevier/Academic Press 2013; pp. 313-342.

22. Radomski MW, Palmer RM, Moncada S. The anti-aggregating properties of vascular endothelium: Interactions between prostacyclin and nitric oxide. Br J Pharmacol 1987;92:639646.

23. Storrie B, Madden EA. Isolation of subcellular organelles. Methods Enzymol 1990;182:203-225.

24. Israels SJ, McMillan EM, Robertson C, Singhory S, McNicol A. The lysosomal granule membrane protein, lamp-2, is also present in platelet dense granule membranes. Thromb Haemost 1996;75:623-629.

25. Nishibori M, Cham B, McNicol A, Shalev A, Jain N, Gerrard JM. The protein cd63 is in platelet dense granules, is deficient in a patient with hermansky-pudlak syndrome, and appears identical to granulophysin. J Clin Invest 1993;91:1775-1782.

26. Holmsen H, Dangelmaier CA, Rongved S. Tight coupling of thrombin-induced acid hydrolase secretion and phosphatidate synthesis to receptor occupancy in human platelets. Biochem J 1984;222:157-167.

27. Vorland M, Holmsen H. Phospholipase d in human platelets: Presence of isoenzymes and participation of autocrine stimulation during thrombin activation. Platelets 2008;19:211224.

28. Kim S, Foster C, Lecchi A, Quinton TM, Prosser DM, Jin J, Cattaneo M, Kunapuli SP. Protease-activated receptors 1 and 4 do not stimulate g(i) signaling pathways in the absence of secreted adp and cause human platelet aggregation independently of g(i) signaling. Blood 2002;99:3629-3636.

29. Li Z, Delaney MK, O'Brien KA, Du X. Signaling during platelet adhesion and activation. Arterioscler Thromb Vasc Biol 2010;30:2341-2349.

30. Silverstein RL, Febbraio M. Identification of lysosome-associated membrane protein2 as an activation-dependent platelet surface glycoprotein. Blood 1992;80:1470-1475.

31. Chronos NA, Wilson DJ, Janes SL, Hutton RA, Buller NP, Goodall AH. Aspirin does not affect the flow cytometric detection of fibrinogen binding to, or release of alpha-granules or lysosomes from, human platelets. Clin Sci (Lond) 1994;87:575-580.

32. McKenzie ME, Malinin AI, Bell CR, Dzhanashvili A, Horowitz ED, Oshrine BR, Atar D, Serebruany VL. Aspirin inhibits surface glycoprotein iib/iiia, p-selectin, cd63, and cd107a receptor expression on human platelets. Blood Coagul Fibrinolysis 2003;14:249-253.

33. Ohlmann P, Laugwitz KL, Nurnberg B, Spicher K, Schultz G, Cazenave JP, Gachet C. The human platelet adp receptor activates gi2 proteins. Biochem J 1995;312 ( Pt 3):775-779.

34. Hollopeter G, Jantzen HM, Vincent D, Li G, England L, Ramakrishnan V, Yang RB, Nurden P, Nurden A, Julius D, et al. Identification of the platelet adp receptor targeted by antithrombotic drugs. Nature 2001;409:202-207.

35. Kajikawa N, Kaibuchi K, Matsubara T, Kikkawa U, Takai Y, Nishizuka Y, Itoh K, Tomioka C. A possible role of protein kinase c in signal-induced lysosomal enzyme release. Biochem Biophys Res Commun 1983;116:743-750.

36. Kim-Shapiro DB, Schechter AN, Gladwin MT. Unraveling the reactions of nitric oxide, nitrite, and hemoglobin in physiology and therapeutics. Arterioscler Thromb Vasc Biol 2006;26:697-705.

37. Rao GH, Krishnamurthi S, Raij L, White JG. Influence of nitric oxide on agonistmediated calcium mobilization in platelets. Biochem Med Metab Biol 1990;43:271-275. 


\section{Figure legends}

Figure 1. Thrombin causes dose dependent exocytosis of lysosomes in human platelets.

(A) Isolated platelets $\left(3 \cdot 10^{8}\right.$ platelets $\left./ \mathrm{ml}\right)$ were treated with thrombin $(0.1,0.3,1$ and $5 \mathrm{U} / \mathrm{ml})$ for 5 min and, after centrifugation, NAG activity was analysed in the supernatant. (B) Analysis of time-dependent release of NAG after stimulation with $1 \mathrm{U} / \mathrm{ml}$ of thrombin using the same experimental system as in (A). (C) Platelets in diluted whole blood were exposed to thrombin for 1 or 10 min and lysosomal exocytosis was determined by flow cytometry as the fraction of platelets binding anti-LAMP-1. Data are expressed as means \pm SEM, (A: $n=4, B: n=8, C: n=6)$. For statistical analysis one-way ANOVA (A and B) or repeated measures ANOVA (C) was followed by Dunnett's multiple comparison tests against the control $(0 \mathrm{U} / \mathrm{ml})$. Significant differences are indicated by ${ }^{*} \mathrm{p}<0.05,{ }^{* *} \mathrm{p}<0.01, * * * \mathrm{p}<0.001$ compared to control. In $\mathrm{C}$, surface expression after $1 \mathrm{~min}$ vs. $10 \mathrm{~min}$ was compared using paired t-test. Significant differences are indicated by \# $\mathrm{p}<0.05$, \#\# $\mathrm{p}<0.01$, \#\#\# $\mathrm{p}<0.001$.

\section{Figure 2. PAR1 and PAR4 activation induce lysosomal exocytosis in human platelets.}

Platelets in diluted whole blood were stimulated for 10 minutes with low, medium and high concentration of PAR1-AP (2.5, 5 or $30 \mu \mathrm{M})$ and PAR4-AP (25, 50 or $150 \mu \mathrm{M})$. Following incubation with anti-LAMP-1, the LAMP-1 positive platelet fraction was analysed by flow cytometry. Data is expressed as means \pm SEM, $(n=5)$. Samples were compared to control using repeated measures ANOVA followed by Dunnett's multiple comparison tests, significant differences are indicated by ${ }^{*} \mathrm{p}<0.05,{ }^{* *} \mathrm{p}<0.01,{ }^{* * *} \mathrm{p}<0.001$. Surface expression of LAMP1 upon PAR1-AP and/or PAR4-AP stimulation was compared, for low, medium and high concentrations respectively, using repeated measures ANOVA followed by Bonferroni's multiple comparison tests, significant differences are indicated by $\# \mathrm{p}<0.05$, \#\# $\mathrm{p}<0.01$, \#\#\# $\mathrm{p}<0.001$. 
Figure 3. Cangrelor, but not ASA, prevents thrombin-induced lysosomal exocytosis from human platelets.

Platelets were incubated with ASA (100 $\mu \mathrm{M}, 15 \mathrm{~min})$ and cangrelor (5 $\mu \mathrm{M}, 5 \mathrm{~min})$ alone or in combination and then stimulated with thrombin $(0.3$ or $1 \mathrm{U} / \mathrm{ml})$. (A) Isolated platelets $\left(3 \cdot 10^{8}\right.$ platelets/ml) were stimulated with thrombin for $1 \mathrm{~min}$ and NAG activity was analysed in the supernatant. (B) Platelets in diluted whole blood were stimulated with thrombin for 1 min after which the fraction of LAMP-1 positive platelets was determined using flow cytometry. (C) Maximal platelet aggregation and (D) ATP-secretion analysed in isolated platelets stimulated with thrombin. (E) Platelets in diluted whole blood were stimulated with thrombin for $1 \mathrm{~min}$ and P-selectin expression was determined using flow cytometry. Data are expressed as means \pm SEM (A, B and E: n=5, C-D: n=3). For statistical analysis one-way ANOVA (in A, C and D) and repeated measures ANOVA (in B and E) followed by Dunnett's multiple comparison tests was used for statistical analysis. Significant differences are indicated by ${ }^{*} \mathrm{p}<0.05,{ }^{* *} \mathrm{p}<0.01$, $* * * \mathrm{p}<0.001$.

Figure 4. SNAP and PGI 2 prevent thrombin-induced lysosomal exocytosis from human platelets.

Platelets were incubated with the NO-donor SNAP (10 $\mu \mathrm{M}, 2$ min) and PGI 2 (1 $\mu \mathrm{M}, 2$ min) alone or in combination and stimulated with thrombin $(0.3$ or $1 \mathrm{U} / \mathrm{ml})$. (A) Isolated platelets $\left(3 \cdot 10^{8}\right.$ platelets $\left./ \mathrm{ml}\right)$ were stimulated with thrombin for $1 \mathrm{~min}$ and NAG activity was analysed in the supernatant. (B) Platelets in diluted whole blood were stimulated with thrombin (1 min) and the fraction of LAMP-1 positive platelets was determined using flow cytometry. (C) The same experiment as in B performed in PRP. (D) Maximal platelet aggregation and (E) ATPsecretion analysed in isolated platelets stimulated with thrombin. (F) Platelets in diluted whole 
blood were stimulated with thrombin $(1 \mathrm{~min})$ and the fraction of P-selectin positive platelets was determined using flow cytometry. (G) The same experiment as in F performed in PRP. Data are expressed as means \pm SEM (A, B, C, F and G: n=5, D-E: n=3). For statistical analysis one-way ANOVA (A, D and E) and repeated measures ANOVA (B, C, F and G) was followed by Dunnett's multiple comparison tests against the respective control. Significant differences are indicated by $* \mathrm{p}<0.05, * * \mathrm{p}<0.01, * * * \mathrm{p}<0.001$.

\section{Supplementary figure S1. Release of $\alpha$-granule by stimulation with PAR1-AP and PAR4-} AP.

Heparinized whole blood was diluted (1:12) in HEPES buffer containing agonists, anti-GPIIb and anti-P-selectin. Samples were incubated for 10 minutes at room temperature, diluted 1:20 in HEPES buffer and then analysed on a Coulter Epics XL.MCL flow cytometer (Beckman Coulter). The agonist concentrations shown are chosen to induce approximately 20\% (low), $50 \%$ (medium) or $100 \%$ (high) release of $\alpha$-granules (percentage of P-selectin-positive platelets). Data is expressed as means \pm SEM, $(n=5)$.

Supplementary figure S2. Stimulation with PAR1-AP and PAR4-AP for 55 min induces lysosomal exocytosis in a fraction of human platelets.

Platelets in diluted whole blood were stimulated for 55 minutes in room temperature with low, medium and high concentration of PAR1-AP (2.5, 5 or $30 \mu \mathrm{M})$ and PAR4-AP (25, 50 or 150 $\mu \mathrm{M})$. After incubation with anti-LAMP-1, the fraction of platelets exposing LAMP-1 on the surface was analysed by flow cytometry. Data is expressed as means \pm SEM, $(n=5)$. Samples were compared to control using repeated measures ANOVA followed by Dunnett's multiple comparison tests, significant differences are indicated by ${ }^{*} \mathrm{p}<0.05,{ }^{* *} \mathrm{p}<0.01,{ }^{* * *} \mathrm{p}<0.001$. Surface expression of LAMP-1 upon PAR1-AP and/or PAR4-AP stimulation was compared, 
for low, medium and high concentrations respectively, using repeated measures ANOVA followed by Bonferroni’s multiple comparison tests, significant differences are indicated by \# $\mathrm{p}<0.05, \# \# \mathrm{p}<0.01, \# \# \# \mathrm{p}<0.001$

\section{Supplementary figure S3. The effect of cangrelor on thrombin-induced lysosomal exocytosis from human platelets is maintained at longer stimulation.}

Platelets were incubated with ASA (100 $\mu \mathrm{M}, 15 \mathrm{~min})$ and cangrelor (5 $\mu \mathrm{M}, 5 \mathrm{~min})$ alone or in combination and then stimulated with thrombin $(0.3$ or $1 \mathrm{U} / \mathrm{ml})$. (A) Isolated platelets $\left(3 \cdot 10^{8}\right.$ platelets/ml) were stimulated with thrombin for 5 min and NAG activity was analysed in the supernatant. (B-C) Platelets in diluted whole blood were stimulated with thrombin for $10 \mathrm{~min}$ after which the fraction of (B) LAMP-1 and (C) P-selectin positive platelets was determined using flow cytometry. Data are expressed as means \pm SEM $(n=5)$. For statistical analysis repeated measures ANOVA, followed by Dunnett's multiple comparison tests against the respective control was used. Significant differences are indicated by $* \mathrm{p}<0.05$, ${ }^{* * *} \mathrm{p}<0.001$.

\section{Supplementary figure S4. SNAP prevents thrombin-induced lysosomal and $\alpha$-granule exocytosis from human platelets in platelet-rich plasma but not in diluted whole blood.} Platelets were incubated with the NO-donor SNAP (10 or $30 \mu \mathrm{M}, 2$ min) and stimulated with thrombin ( 0.3 or $1 \mathrm{U} / \mathrm{ml}, 1 \mathrm{~min})$. (A-B) The fraction of platelets positive for LAMP-1 in diluted whole blood (A) or platelet-rich plasma (PRP) (B) determined using flow cytometry. (C-D) P-selectin expression on platelets in diluted whole blood (C) or PRP (D) determined using flow cytometry. Data are expressed as means \pm SEM (n=5). For statistical analysis repeated measures ANOVA was followed by Dunnett's multiple comparison tests against the respective control. Significant differences are indicated by ${ }^{*} \mathrm{p}<0.05$, ${ }^{* * *} \mathrm{p}<0.001$. 
Supplementary figure S5. SNAP- and PGI2-prevented thrombin-induced lysosomal exocytosis from human platelets is maintained at longer stimulation.

Platelets were incubated with the NO-donor SNAP (10 $\mu \mathrm{M}, 2$ min) and PGI 2 (1 $\mu \mathrm{M}, 2$ min) alone or in combination and stimulated with thrombin $(0.3$ or $1 \mathrm{U} / \mathrm{ml})$. (A) Isolated platelets $\left(3 \cdot 10^{8}\right.$ platelets $\left./ \mathrm{ml}\right)$ were stimulated with thrombin for 5 min and NAG activity was analysed in the supernatant. (B) Platelets in diluted whole blood were stimulated with thrombin (10 min) and the fraction of LAMP-1 positive platelets was determined using flow cytometry. (C) The same experiment as in B performed in PRP. (D) Platelets in diluted whole blood were stimulated with thrombin (10 min) and the fraction of P-selectin positive platelets was determined using flow cytometry. (E) The same experiment as in D performed in PRP. Data are expressed as means \pm SEM $(n=5)$. For statistical analysis repeated measures ANOVA was followed by Dunnett's multiple comparison tests against the respective control. Significant differences are indicated by ${ }^{*} \mathrm{p}<0.05, * * \mathrm{p}<0.01, * * * \mathrm{p}<0.001$. 